\title{
Procalcitonin as A Predictor of Left Ventricular Systolic Dysfunction in Sepsis Patients
}

\author{
Trisulo Wasyanto'), Ahmad Yasa'), Bhisma Murti²) \\ 1)Department of Cardiology and Vascular Medicine, Faculty of Medicine, \\ Universitas Sebelas Maret/Dr. Moewardi General Hospital, Surakarta \\ ${ }^{2)}$ Masters Program in Public Health, Universitas Sebelas Maret, Surakarta
}

\begin{abstract}
Background: Release of cytokines in sepsis can cause left heart failure and left ventricular systolic dysfunction (LVSD). In sepsis, there is a releasing of Procalcitonin (PCT) and tumor necrosis factor- $\alpha$ (TNF- $\alpha$ ) because of the stimulation of cytokine pro inflammation effected by activated NF$\mathrm{kB}$. This study aimed to examine PCT as a predictor of LVSD in sepsis, value of Area Under the curve (AUC), sensitivity, specificity, cut off point, and probability of PCT and TNF- $\alpha$ as a predictor of LVSD, and then to identify the best diagnostic predictor of LVSD.

Subjects and Method: This was a cross-sectional study. A sample of 71 sepsis patients aged $>18$ years old admitted to the intensive care unit (ICU) from November 2016 to March 2017was selected for this study. The dependent variable was left ventricle ejection fraction (LVEF). The independent variables were PCT and TNF- $\alpha$. LVEF and diastolyc function were measured by transthoracicechocardiography. Data on PCT and TNF- $\alpha$ level were obtained from medical record.

Results: As many as 22 patients had mild sepsis (30.9\%), 40 patients had severe sepsis (56.4\%), and 9 patients had septic shock (12.7\%). The AUC value of PCT level was 0.81 (95\% CI 0.71-0.91; $\mathrm{p}<0.001$ ). Optimal cut off point was $\geq 7.88 \mathrm{ng} / \mathrm{mL}$ and Diagnostic Odd Ratio (DOR) was 5.55. The AUC value of TNF- $\alpha$ level was 0.73 (95\% CI 0.60-0.86; $p=0.002$ ). Optimal cut off point was $\geq 7.36$ $\mathrm{pg} / \mathrm{ml}$ and DOR was 5.03. Multivariate analysis was resulted that PCT was the best predictor of LVSD (AUC 0.70), and TNF- $\alpha$ (0.69). The combination of PCT + TNFa would increase diagnostic value with AUC 0.77.

Conclusion: PCT was a better predictor of LVSD than TNF- $\alpha$. This finding is significant, since it will enable clinicians to easily diagnose LVSD by measuring PCT. The combination of PCT and TNFa was the best efficient diagnostic predictor of LVSD.
\end{abstract}

Keywords: Left Ventricular Systolic Dysfunction, predictor , PCT, TNF- $\alpha$

\section{Correspondence:}

Trisulo Wasyanto. Department of Cardiology and Vascular Medicine, Faculty of Medicine, Universitas Sebelas Maret. Jl. Ir. Sutami 36A, Surakarta 57126, Central Java. Email: trisulo.wasyanto@gmail.com.

\section{BACKGROUND}

Sepsis still becomes health problem since this causes a high mortality and morbidity rate. Sepsis may be associated with concurrent left ventricular systolic dysfunction (LVSD) and patients who develop LVSD during sepsis may have a worse prognosis than patients who do not. The release of local cytokine products along with proinflammatory cytokines, excessive nitricoxide and cytosolic calcium, can lead to mitochondrial dysfunction and cell apoptosis. In severe sepsis, tumor necrosis factor$\alpha$ (TNF- $\alpha$ ) elevated and will cause abnormalities in theheartandliver. In the heart, TNF- $\alpha$ will cause myocardial dysfunction. In the liver, TNF- $\alpha$ will stimulate procalcitonin (PCT) secretion, that isa microbial infection biomarker. These cellular events can lead to myocardial dysfunction characterized as LVSD (Flierl et al, 2008 and Fernandes, 2012). To diagnose LVSD, clini- 
cians should use an echocardiogram. Unfortunately, not all hospitals in Indonesia have echocardiogram machines. The identification of predictors LVSD inseptic patients is expected to decrease morbidity and mortality rates. Until now, no specific predictors have been available to detect LVSD. The diagnosis of LVSD may be possible in a peripheral hospitalby simply measuring PCT or TNF- $\alpha$ levels in serum.

Pattern recognition receptors (PRRs) consisting of a toll-ikereceptor (TLR) and CD14 on the surface of monocytes can recognize pathogen associated molecular patterns (PAMPs) that enter the body. The interaction between PAMPs and PRRs on the surface of monocytes results in the activation of pro-inflammatory cytokines (TNF- $\dot{\alpha}$, interleukin [IL]-1 $\beta$ and IL-6) and anti-inflammatory cytokines (IL-4,IL-5, IL6, and IL-10). Lipopolysaccharide (LPS) binds with the CD14 receptor and TLR-4 on the monocyte surface and activates intra cellular signal transduction involving various adaptor proteins such as: myeloid differentiation protein88 (MyD88), TNF receptor associated factor (TRAF), and kinase enzyme-likeinhibitor kappa b kinase (IKK). These cellular conditions activate a transcription protein called nuclear factor kappa $\mathrm{B}$ (NF-kB). NF-kB is a transcription protein in the cytoplasm activated by binding fragmentation within hibitor kappa b (IKB), which enters the nucleus of monocytes and initiates RNA transcription of pro-and antiinflammatory cytokine molecules (Guntur, 2001; Kibe et al, 2011; Celes et al, 2013).

Under normal circumstances, TNF- $\alpha$ levels are almost undetectable in the blood, and peak concentrations rise within $2 \mathrm{~h}$ if there is sepsis. Since TNF- $\alpha$ has a very short half-life (approximately $18.2 \mathrm{~min}$ ) and is unstable, it has rarely been used as a sepsis biomarker (Oliver et al, 1993).
This study aimed to prove PCT as a predictor of LVSD in sepsis, to search a value of Area Under the Curve (AUC), sensitivity, specificity, cut off point and probability PCT and TNF- $\alpha$ as a predictor of LVSD in sepsis patients, to search the best diagnostic predictor of LVSD in sepsis patients.

\section{SUBJECTS AND METHOD}

This was a cross-sectional study carried out at the tertiary intensive care unit (ICU) in Dr. Moewardi Hospital, Surakarta, Central Java, Indonesia, from November 2016 to March 2017. A sample of 71 patients aged over 18 years old was selected for this study by simple random sampling.

We chose patients with sepsis as defined by the American College of Chest Physicians/Society of Critical Care Medicine consensus criteria (Delinger et al, 2008). The exclusion criteria were as follows: all sepsis cases with ischemic heart disease, heart failure, chronic kidney disease or valvular heart disease. This study was approved by the Ethical Review Committee from Universitas Sebelas Maret /Dr. Moewardi Hospital and informed consent was obtained from the patients or their legal representatives.

Using a formula based on diagnostic research and the area under the curve (AUC) output, the sample size for this study was at least 68 and we analyzed 71 patients. The variables evaluated by the researchers included: LVEF, PCT, and TNF- $\alpha$. Serum samples taken to measure PCT and TNF- $\alpha$ were centrifuged within $45 \mathrm{~min}$ after collection. The resulting plasma was frozen at $-20^{\circ} \mathrm{C}$ until it was ready to be analyzedin a blinded fashion. PCT was analyzed with electrochemiluminescence using an enzyme-linked fluorescent assay method (VIDAS equipment and Brahms reagents). The normal value of PCT was $<0.05 \mathrm{ng} / \mathrm{mL}$. 
TNF- $\alpha$ was measured by applying a quantitatives and which enzyme immuno-assay with human TNF- $\alpha /$ TNFSF1AHS (R\&D systemsInc., Minneapolis, MN, USA); the reagent kit used for human TNF- $\alpha$ was also from R\&D Systems. The immune-assay technique using human TNF- $\alpha$ was conducted with a Microplate Reader 680 series device. The normal value of TNF- $\alpha$ was 0.5 pg/mL Transthoracic echocardiography performed by 2 cardiologists to determine left ventricular systolic function (LVSF) and LVEF using the Simpson method (De Backer, 2011 and Griffey et al, 2010).

Diastolic function was assessed by measuring $\mathrm{E}$ and A peak velocities using a spectral Doppler to measure mitral in flow. LVSD was defined as LVEF $\leq 45 \%$ (De Backer, 2011). Diastolic dysfunction was defined as an E/A ratio $<1$ with a deceleration time $(\mathrm{DcT})>240 \mathrm{~ms}$ or $\mathrm{E} / \mathrm{A}$ ratio $>2$ with DcT <160ms (Griffey et al, 2010). Data were analyzed with a $2 \times 2$ table and a receiver operating characteristic (ROC) curve using SPSS 22 for Windows. Analysis with $2 \times 2$ tables was used to determine the sensitivity, specificity, and probability of PCT and TNF- $\alpha$ as LVSD predictors in septic patients. ROC curve analysis to determine the AUC and optimal cut-off points for PCT and TNF levels were determined in septic patients who developed LVSD.

\section{RESULTS}

This study was conducted from November 2016 to march 2017 in tertiary ICU at Moewardi Hospital Surakarta Indonesia. Research subjects consisted of 71 patients divided into 22 mild sepsis (30.9\%), 40 severe sepsis (56.4\%) and 9 septic shock (12.7\%). There were 69 patients having systolic dysfunction (97.2\%), meanwhile there were 48 patients having left ventricular systolic dysfunction (67.6\%).

Table 1. Basic characteristic of the study participants

\begin{tabular}{lccc}
\hline Variabel & $\begin{array}{c}\text { LVSD } \\
(\mathbf{n}=\mathbf{4 8})\end{array}$ & $\begin{array}{c}\text { Non LVSD } \\
(\mathbf{n}=\mathbf{2 3})\end{array}$ & $\mathbf{p}$ \\
\hline Gender: & & & \\
Male & $18(37.5 \%)$ & $8(34.8 \%)$ & 0.520 \\
Female & $30(62.5 \%)$ & $15(65.2 \%)$ & 0.160 \\
Age (Years) & $57.2 \pm 15.3$ & $51.5 \pm 16.7$ & \\
Elderly: & & & 0.324 \\
$\quad$ Non-elderly & $27(56.3 \%)$ & $15(65.2 \%)$ & \\
Elderly & $21(43.8 \%)$ & $8(34.8 \%)$ & $<0.001$ \\
Sepsis Severity: & & & \\
Mild Sepsis & $0(0.0 \%)$ & $22(95.7 \%)$ & $1(4.3 \%)$ \\
$\quad$ Severe Sepsis & $39(81.3 \%)$ & $0(0.0 \%)$ & \\
Septic shock & $9(18.8 \%)$ &
\end{tabular}

Abbreviations- LVSD: left ventricular systolic dysfunction

The sepsis were grouped based on LVSD. This characteristic showed that there were not differences in gender $(\mathrm{p}=0.520)$, age $(\mathrm{p}=0.160)$ in both elderly (>6oyears old) and non-elderly $(\mathrm{p}=0.324)$. First, there was a different characteristic for patients ofLVSD compared to non LVSD based on sepsis degree $(\mathrm{p}<001)$. 
The AUC value of PCT level was 0.81 (95\% CI 0.71-0.91), p<0.001. Optimal cut off point was $\geq 7.88 \mathrm{ng} / \mathrm{mL}$ with sensitivity $70.8 \%$ and specificity $69.6 \%$, and Diag- nostic Odd Ratio (DOR) was 5.55 (95\% CI 1.88-16.42) with PCT probability $\geq 7.88$ $\mathrm{ng} / \mathrm{mL}$. Next, the occurrence of left ventricular systolic dysfunction was $84.7 \%$.

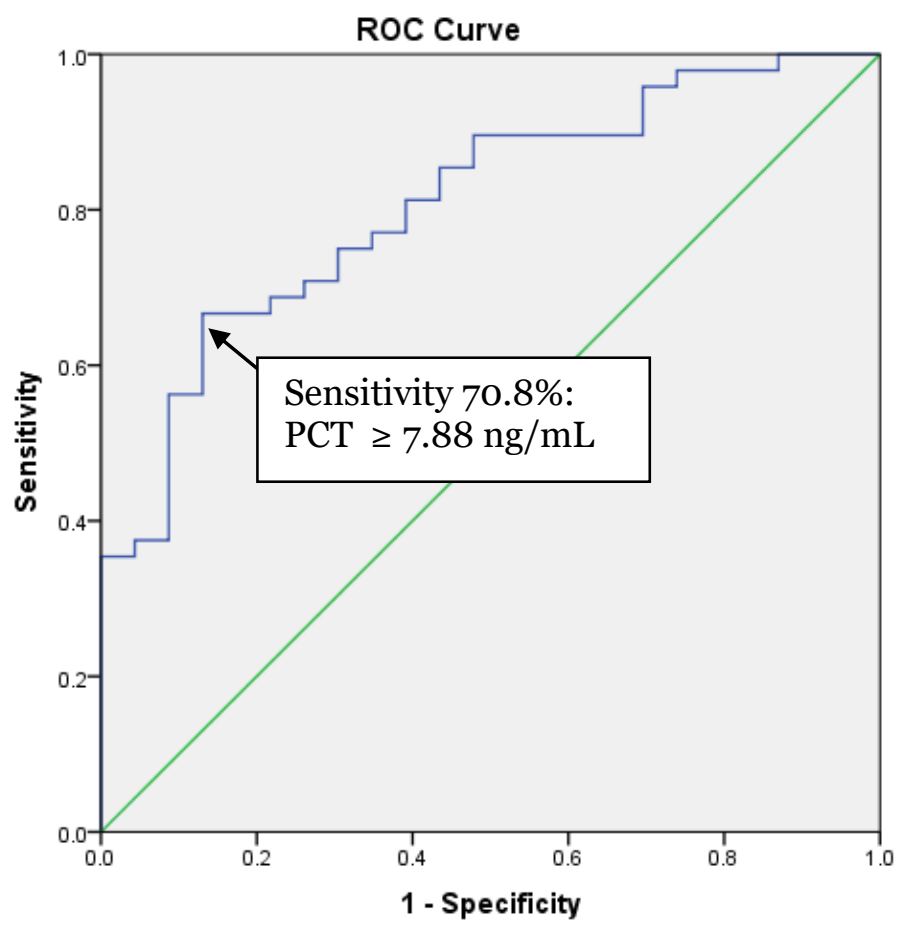

Figure 1. ROC curve diagnostic accuracy of PCT level to diagnose LVSD in sepsis patients. Width of ROC curve o.81 (0.71-0.91)

Table 2. Comparison of area under the curve in PCT and TNF-a for LVSD

\begin{tabular}{lcccc}
\hline Cut off variable & $\begin{array}{c}\text { Area under the } \\
\text { curve (AUC) }\end{array}$ & $\mathbf{p}$ & Lower limit & Upper limit \\
\cline { 4 - 5 } & 0.81 & $<0.001$ & 0.71 & 0.91 \\
TNF & 0.73 & 0.002 & 0.60 & 0.86 \\
\hline
\end{tabular}

The AUC value of TNF- $\alpha$ level was 0.73 (95\% CI 0.60 to $0.86 ; \mathrm{p}=0.002$ ). Optimal cut off point was $\geq 7.36 \mathrm{pg} / \mathrm{ml}$ with sensitivity $68.8 \%$, and specificity $69.6 \%$. Diagnostic Odd Ratio (DOR) was 5.03 with TNF- $\alpha$ probability $\geq 7.36 \mathrm{pg} / \mathrm{ml}$, and left ventricular systolic dysfunction occurrence was $83.4 \%$.

Bivariate analysis of LVSD and factors such as age, severity of sepsis, levels of PCT and TNF- $\alpha$ showed that PCT and
TNF- $\alpha$ had a value of $\mathrm{p}<0.25$ and these variables were included in multivariate analysis. Multivariate analysis indicated that PCT was good with an AUC of 0.70, sensitivity of $70.8 \%$ and specificity of $69.6 \%$. The predicted value of TNF- $\alpha$ was quite good with an AUC of 0.69 , sensitivity of $68.8 \%$ and specificity of $69.6 \%$. The combination of PCT + TNF- $\alpha$ would increase diagnostic value with AUC 0.77 (see Table 3). 


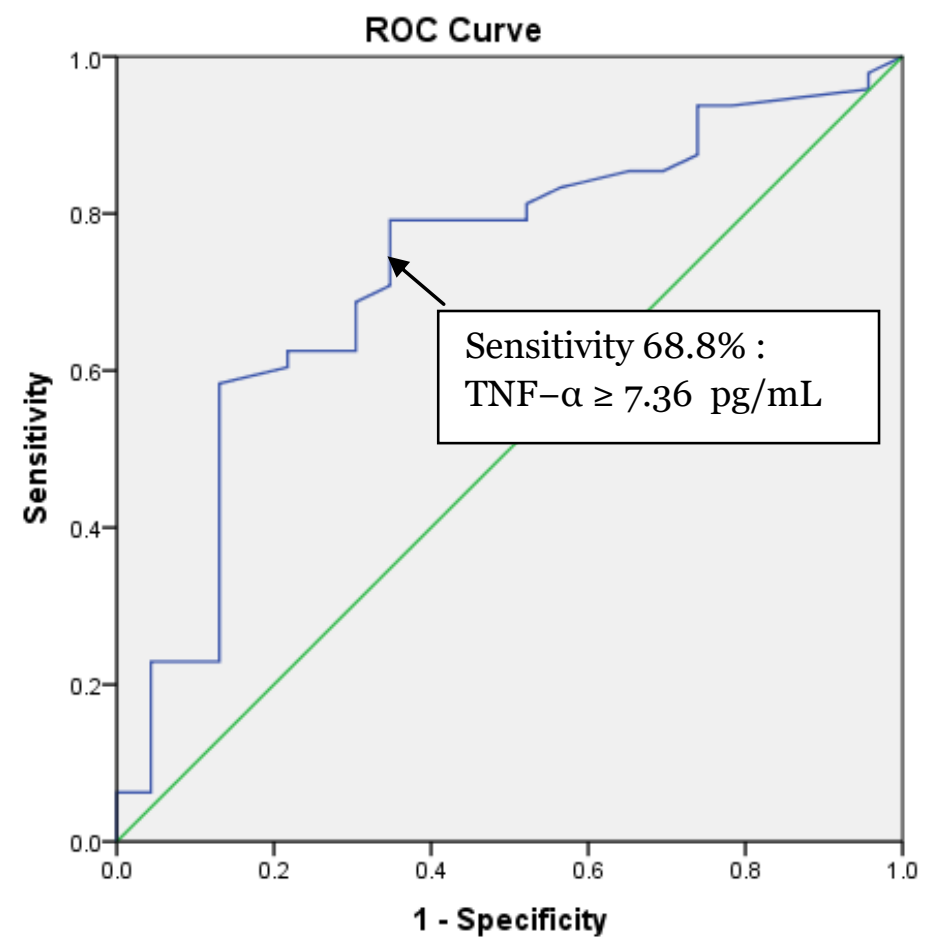

Diagonal segments are produced by ties.

Figure 2. ROC curve diagnostic accuracy of TNF-a level to diagnose LVSD in sepsis patients. Width of ROC curve o.73(0.60-0.86)

Table 3. Multivariate analysis in both the comparison among AUC of PCT, TNF-a and the combination of PCT + TNF-a as a predictor of LVSD in sepsis patients

\begin{tabular}{lcccc}
\hline \multirow{2}{*}{ Cut off variable } & \multirow{2}{*}{ AUC } & \multicolumn{2}{c}{ 95\% CI } & p \\
\cline { 3 - 4 } PCT & 0.70 & 0.57 & 0.83 & 0.006 \\
TNF- $\alpha$ & 0.69 & 0.56 & 0.83 & 0.009 \\
PCT + TNF- $\alpha$ & 0.77 & 0.64 & 0.90 & $<0.001$ \\
\hline
\end{tabular}

\section{DISCUSSION}

This study aimed to demonstrate the efficacy of PCT and TNF- $\alpha$ as a predictors of LVSD in sepsis, and to identify an AUC, sensitivity, specificity, optimal cut-off point and probability for PCT and TNF- $\alpha$ as predictors of LVSD in sepsis. Furthermore, our goal was to identify for the best diagnostic predictor of LVSD in sepsis. To identify complications of LVSD (left heart failure) in sepsis, cardiologists should use echocardiography, which requires special skills and devices, and is not always avail- able in health-care facilities, especially in peripheral hospitals. In addition, it is sometimes verydifficultto perform echocardiography in patients who are not "echogenic", including those who are obese, those with chronic obstructive pulmonary disease (COPD), or patients in the ICU who are attached to various life support devices (Griffee et al, 2010). The results of this study are expected to make it easier to diagnose septic patients with LVSD, simply by checking the serum levels of PCT or TNF- $\alpha$ (without using an echocardiogram). 
This study will have clinical applicability in peripheral hospitals (hospital types C or D in Indonesia). Those with severe sepsis, exhibit hemodynamic changes and hypoperfusion in some organs. With worsening sepsis, the ability of cardiac muscle to contrac will decrease, leading to cardiac dilatation and decreased stroke volume, ejection fraction and systemic vascular resistance; vasodilation of blood vessel and hypotension will worsen until septic shock occurs 6.13. Echocardiography has an advantage over invasive examinations because it can be done at the bedside and can easily be done repeatedly without causing the patientharm (Griffee et al, 2010 and De Backer, 2011).

In severe sepsis, there was haemodynamic changing and hypoperfusion of tissue in some organs. Then, multiple organ dysfunction syndrome (MODS) and septic shock would occur. Septic shock in adults refers to a state of acute circulatory failure characterized by persistent arterial hypotension unexplained by other causes. The chronicle of MODS consisted of respiratory failure, liver and intestinal failure, and renal failure. Haematologic and myocardial failure was the last manifestation of MODS, whereas SSP failure could occur in the beginning or in the last of disease step (Pinsky, 2012).

The recent issues mentioned that sepsis complication into heart was signed by myocardial depression and heart index reduction. Myocardial depression in the beginning of sepsis was still reversible in 710 days, however, if sepsis was getting serious, the worse condition would change into irreversible because of septic shock and patients could die (Cavazzoni et al, 2010 and Jozwiak et al, 2011).

This was the first diagnostic study to search a main predictor taken from variables of PCT, and TNF- $\alpha$ in sepsis pati- ents to diagnose LVSD. There were 97. $2 \%$ of sepsis patients having diastolic dysfunction, and this meant that severe sepsis occurred. Therefore, aggressive liquid resuscitation had to be given to those patients. This research proved that PCT could be used as a predictorof LVSD in severe sepsis patients. PCT level was $\geq 7.88$ $\mathrm{ng} / \mathrm{mL}$ and this could be used to diagnose sepsis patients having LVSD with sensitivity $70.8 \%$ and specificity $69,6 \%$. DOR was 5.55 with probability of sepsis patients having LVSD and increasing PCT level occurred $84.7 \%$.

The results of this study are in line with Liu et al. (2013), which indicated that when PCT levels were $>\mathbf{2 n g} / \mathrm{mL}$, a patient may progress toward severe sepsis or septic shock. In addition, it is important to monitor patients with a PCT $>2 \mathrm{ng} / \mathrm{mL}$, and antibiotics should be considered. If PCT levels are very high $(>10 n g / \mathrm{mL})$ the risk of death is higher, as high PCT levels are significantly associated with septic shock, multiple organ dysfunction syndrome and poor outcomes.

In addition, $\mathrm{TNF}-\alpha$ level was $\geq 7.36$ $\mathrm{pg} / \mathrm{ml}$, and the patients were diagnosed as sepsis patients who had LVSD with sensitivity $68.8 \%$ and specificity $69.6 \%$.

Multivariate analysis used ROC curve method and found the use of single predictor as LVSD predictor with AUC value of PCT (0.70). This result was better compared to TNF- $\alpha$ (0.69) with sensitivity $70.8 \%$ and specificity $69.6 \%$. The use of double biomarker as a predictor of LVSD showed that AUC value of PCT + TNF- $\alpha$ (0.77) was better compare to PCT alone (0.70) with sensitivity $70.8 \%$ and specificity $69.6 \%$.

Based on the results of this study, it can conclude that PCT and TNF- $\alpha$ could be used as LVSD predictor for sepsis patients, with AUC 0.81 and 0.73, respectively. 
REFERENCES

Cavazzoni SLZ, Guglielmi M, Parillo JE et al, (2010). Ventricular dilatation is associated with improved cardiovascular performance and survival in sepsis. Chest. 138(4):848-855.

Celes MRN, Prado CM, Rossi MA (2013). Sepsis: Going to the heart of the matter. Pathobiology. 80:70-86. Doi: 10.1159/000341640.

De Backer D, Baron AV (2011). Hemodynamic monitoring using echocardiography in the critically ill, Septic Shock. Doi: 10.1007/978-3-540-87956-5_11.

Dellinger RP, Levy MM, Carlet JM, et al. (2008). Surviving sepsis campaign: international guidelines for management of severe sepsis and septic shock. Crit Care Med. 36:296-327. Doi: 10.1097/01.CCM. 0000298158.12101.41.

Fernandes CJ, Santucci CAM (2012). Review article: Myocardial dysfunction in sepsis: a large, unsolved puzzle. Critical Care Research and Practice. Doi: 10.1155/2012/896430.

Flierl MA, Rittirsch D, Huber-Lang MS et al, (2008). Molecular events in the cardiomyopathy of sepsis. Mol Med. 14(5-6): 327-336.

De Backer MJ, Merkel MJ, Wei KS (2010). The role of echocardiography in hemodynamic assessment of septic shock. Crit Care Clin 26: 365-382.

Griffee MJ, Merkel MJ, Wei KS (2010). The role of echocardiography in hemodynamic assessment of septic shock. Crit Care Clin. 26: 365-382. DOI: 10.1016/j.ccc.2010.01.001.

Guntur H (2001). Differences in immune responses and its role in sepsis and septic shock, an approach imunopathobiologic sepsis and septic shock in immunocompromise and non immunocompromise. Dissertation. Airlangga University Graduate Program.

Jozwiak M, Percichini R, Monnet X et al, (2011). Management of myocardial dysfunction in severe sepsis. Semin Respir Crit Care Med. 32(2): 206-214.

Kibe S, Adams K, Barlow G (2011). Diagnostic and prognostic biomarker of sepsis in critical care. J Antimicrob Chemother. 66(2):ii33-ii40.

Lipinska-Gedica M, Mierzchala M (2012). Pro-atrial natriuretic peptide (proANP) level in patients with severe sepsis and septic shock: prognostic and diagnostic significance. Infection. 40:303-309

Liu YJ, Du P, Rao J (2013). Procalcitonin as a diagnostic and prognosticmarker for sepsiscaused by intestinalinfection: a case report. Eur Rev Med Pharmacol Sci. 17(10):1311-3.

Oliver JC, Bland LA, Oettinger CW et al, (1993). Cytokine kinetics in an invitro whole blood model following an endotoxin challenge. Lymphokine Cytokine Res. 12(2):115-120.

Pinsky MR, 2012. Septic Shock . E medicine Journal 13(8): 1-21. 